\title{
Standardizing Canadian Decision Support Systems
}

\section{Keith Jansa*}

Canada Health \& Medical Informatics, Ottawa, Canada

Adverse events undeniably compromise patient safety. Inadvertent complications attributable to preventable error rather than patient illness or disease suggests a fragmented and dire system of care. Adverse events by definition refer to diagnostic errors, and make up a substantial fraction of all medical errors leading to unnecessary morbidity, deaths, and healthcare costs. The Canadian Adverse Events Study published in 2004 and conducted by the Canadian Institute of Health Information found that the overall rate of adverse events in 2000 was seven point five per one hundred patients admitted to Canadian hospitals, one point six of which were associated with causing death [1].

"About 1 out of every 10 patients with health problems surveyed in Canada reported that they had been given a wrong medication or a wrong dose in the past two years. Three out of every 20 Canadians surveyed reported an adverse event; about $46 \%$ of these resulted in a serious health problem" [2].

"Close to 37 per cent of adverse events in the study were potentially preventable. Based on this, the researchers estimate there were 70,000 preventable adverse events across the country in 2000" [2].

"About 1 out of every 10 patients with health problems surveyed in Canada reported that they had been given a wrong medication or a wrong dose in the past two years. Three out of every 20 Canadians surveyed reported an adverse event; about $46 \%$ of these resulted in a serious health problem."

Inclusive to adverse events is whether such events occur because of an act of omission where a given diagnostic test, treatment intervention, or surgical procedure is absent from having been done resulting in a medical error; or by an act of commission where health care professionals direct specific care that results in again medical error, or an adverse incident. Outwardly descriptive of the former, "a 29-year-old woman presents to hospital with overwhelming chest pain. She is told that she is too young to be having a heart attack, and a viral cause is presumed. As a result of this 'clinical tunnel vision' she dies of her myocardial infarct" [3]. Similarly, and illustrative of the latter, is where "an 18-month-old boy who has fallen on his head almost dies of a subdural hematoma because the doctor insists, despite his mother's claims to the contrary, that the child is probably suffering from a leg injury, as evidenced by his unsteady stance" [3].

"While adverse events are largely construed as human error that can be characterized as malpractice or negligence, what we now know is that a great majority of incidents may be attributable to what is known as system error. Adverse events are now regarded not so much an issue of negligence and/or liability, but of quality health care and patient safety" [4].

Identifiable as a rather systemic concern, rather than as a caseby-case and individualistic occurrence, adverse events resulting from medical error were able to be summarized into six categories of error: administrative, communicative, diagnostic, documentary, medicinal, and surgical or procedural, respectively [5].

In addition to the categories, ten causal factors were identified in increasing the likelihood of an adverse event to occur, which include: case complexity, discontinuity of care, failure to follow protocol or accepted practice, fatigue, gap in knowledge, high workload, and insufficient information on pharmacological properties of medication, medication side effects, relationship dynamics, and structural problems [5].

"Systemic risks require systemic action. This requires a philosophical change to that of a 'learning culture', where there is an expectation that error will occur every day, that errors need to be anticipated and managed in an atmosphere that promotes and encourages frank discussion and improvement and that offers incentives for healthcare organizations to improve their performance" [6].

"Errors are not isolated problems, but have underlying systemic causes. Research has long shown that working in complex, stressful environments like hospitals makes everyone prone to mistakes. Despite the demand for 'multi-tasking,' the human brain is not capable of keeping more than a few pieces of information straight at any one time. Thus there is a risk of information overload when healthcare professionals must monitor many pieces of equipment in surgery or fill several medication orders in a short time."

To evidently ensure the safety of all Canadians seeking medical attention within the country's hospitals a considerable need exists in standardizing healthcare professional practices. "Standardization is known to decrease the chance of errors because it limits the variety of methods in performing a task" [7]. Likewise, given the current non-standardized approach in healthcare, Canadians are continually subject to unnecessary risks when seeking medical help. Statistically, in 2001 through to 2003 the number of admissions to Canadian hospitals ranged from 3.1 to 3.3 million patients [8] .Given that current statistical evidence suggests that in Canada the number of deaths indicative of an adverse event equals approximately one point six percent is overly suggestive, although a rough estimate, that on average 140 patient each day die in a Canadian hospital because of an adverse event. Moreover, given that current admission rates in Canadian hospitals are approximately 3.2 million patients, on average that translates into a rough 8,767 patients seeking medical attention in Canadian hospitals a day. Although adverse events are a newly researched area of concern, and statistical data has yet to illustrate the distribution of adverse events across Canada, a standardized system of care is still needed to ensure that both patient legal and human rights are maintained. Given, however, that approximately 8,767 patient admittances occur each day and 140 of those are resulting deaths due to adverse events suggests that one-in-every-sixty-third patient admittance will result in the death of

*Corresponding author: Keith Jansa, Canada Health \& Medical Informatics, 1387 Beaucourt Place, Ottawa, Canada, E-mail: kjansa@alumni.uottawa.ca

Received January 12, 2012; Accepted July 25, 2012; Published July 30, 2012

Citation: Jansa K (2012) Standardizing Canadian Decision Support Systems. J Health Med Inform 3:113. doi:10.4172/2157-7420.1000113

Copyright: @ 2012 Jansa K. This is an open-access article distributed under the terms of the Creative Commons Attribution License, which permits unrestricted use, distribution, and reproduction in any medium, provided the original author and source are credited. 
that patient. Are you going to be the sixty-third patient when required to seek immediate medical attention?

\section{Rationale}

The situational relevance to standardize the use of clinical decision support systems is to seemingly limit, if not eliminate, the total number of diagnostic and medicinal adverse events in Canadian hospitals. As such, the Multilevel Approach to Community Health or MATCH planning model should be used, which is "designed to be applied behavioral and environmental risk and protective factors for disease or injury are generally known and when general priorities for action have been determined" [9]. The general focus and explicit goal of operating clinical decision support systems is to improve patient safety in Canadian hospitals, and to ensure that every Canadian patient who seeks medical help within any one of the country's hospitals is subject to the same standardized system of care to improve the quality of care, efficiency of healthcare services, and safety of the patients.

"Decision support systems may also help ensure a minimum standard for quality of care as part of the implementation of practice guidelines by providing patient-specific recommendations after the input of certain clinical variables ... To date no high quality studies have examined the impact of widespread institution of decision support, but local studies suggest that it may be substantial. An evaluation of an antibiotic management system in a single 12-bed Intensive Care Unit over a 3 year period revealed that use of the system resulted in 24 fewer adverse drug reactions and 194 fewer cases of antibioticsusceptibility mismatch. These results and others suggest that national implementation of decision support systems could markedly improve patient safety" [10].

At the organizational level, hospital executives will have an influential part in ensuring the necessary conditions exist to ensure successful implementation of clinical decision support systems. Given that clinical decision support systems standardize differential diagnoses amongst health care professionals, both community leaders and governmental officials will have an influential bearing on success of its implementation. These leaders will need to promote the intervention, and provide any necessary policy amendments to make certain that the Canadian healthcare system has become homogeneous in differentially diagnosing patients.

Inputs into implementing a clinical decision support system are rather simplistic given that the necessary personnel and space resources already exist. Implementation involves improving the healthcare delivery system by integrating a clinical decision support system to the already existing LAN or network computer base in Canadian hospitals. Financially, for example, DXplain is relatively inexpensive considering the billions of dollars allocated by both the provincial and federal governments in Canada and is merely expending a price tag to the Canadian healthcare system that is equivalent to purchasing an operating system for a computer.

The outputs into implementation will involve training healthcare professional in utilizing the clinical decision support system with use of equipment demonstrations. The clinical decision support system will reach each Canadian seeking medical care within Canadian hospitals by providing physicians with differential diagnoses based on the sign and symptoms exhibited by the patients.

Short, intermediate and long-term outcomes will implicate a reduction in differential adverse events in patients who are subject to the intervention, i.e., clinical decision support system. Overall patient satisfaction, improved efficiency in healthcare delivery, and improved quality of care should be noted throughout the length of the intervention. The program length ought to yield at least 6 months, although one year will potentially yield the best results in term of paralleling annual data from prior research conducted on the occurrence of adverse events in Canada.

\section{Target Population}

Although rather broad and all-encompassing, the target population concerns all Canadians who are subject to having to receive medical attention in one of Canada's hospitals. Adverse event evidently span the entire Canadian population, and although current statistical evidence has yet to suggest any particular trends in gender, race, age or socio-economical status in terms of having had been the victim of an adverse event, the only potentially relevant statistic relates to the fact that men receive better hospital care than women [11]. Whether this is suggestive that females experience greater numbers of adverse events is unknown and also even if it were assumed that females are subject to more adverse events, statistical evidence does suggest that women seek medical attention far greater the man anyway.

\section{Stakeholder/Partner Integration}

Given the scope of the implementation clinical decision support systems across all Canadian hospitals, government officials, in particular both MPs and MPPs for the Ministries of Health in each province and territory along with their critic counterparts from all government oppositions' parties ought to be present as stakeholders on a planning committee. Likewise, Canadian hospital executives should be welcome to join in regards to understanding their collaborative role in either piloting or fully implementing a standardized clinical decision support system within their hospitals, and to ensure that all healthcare professionals are integrated, trained, and well-rehearsed on being able to utilize the intervention.

"Governments and other stakeholders should convene an expert committee representing clinical disciplines and management with knowledge of patient safety systems, tools and other resources. This committee would develop an agenda for addressing patient safety issues in Canadian healthcare, including a list of approaches to and sources for methods and tools for patient safety relevant to Canadian health care organizations. This list would be of interest to provincial ministries of health, regional authorities, healthcare organizations and accrediting agencies" [12].

Healthcare professionals, and statistical analysts employed with the Canadian Institute of Health Information should partner with the implementation of a standardized clinical decision support system, and will become a major asset to ensure their role in integrating the already existing the Canadian Medication Incident Reporting and Prevention System, and a new adverse events surveillance system dedicated to identifying medical errors in differential diagnoses. Evidently the participation of technical support will have to be wellversed in troubleshooting the clinical decision support system should it happen to go offline as well as having to possess a supportive role in ensuring that physicians and healthcare professionals are able to utilize the system accurately, without problem, and in complement with their own expertise. The federal government should be the whole contributor to financing Canada's clinical decision support systems and should ensure policy promotes standardizing the way in differentially diagnosing disease and illness in Canada. 


\section{Program Intervention}

The likely and accredited benefits of utilizing clinical decision support systems in hospitals include improving patient safety, bettering the quality of care and advancing the efficiency in health care delivery [13]. Effective execution of clinical decision support system programs across Canadian hospitals will yield reductions in the number of adverse events resulting in disability and/or death in patients. Moreover, the use will generate measurable reductions in the number of occurred adverse drug reactions and will increase the effectiveness of order processing of both medications and diagnostic tests [13]. As well, successful implementation will provide substantial reductions in adverse drug effects, which result in many patient injuries and deaths [14]. The greatest proportion of preventable adverse drug reactions occurs at the drug ordering stage [15]. All medication errors occurring at the ordering stage should be preventable and ideally non-existent. Evidently, with interventional use of a clinical decision support system, healthcare professionals will be able to prescribe pharmaceuticals under a failsafe system. Should the patient's health be at risk because of the prescribe drug, or dose administered, the clinical decision support system identifies the error and suggests a different course of action.

DXplain, a rather modern working example of a clinical decision support system in operational use across various health sector settings in Canada and around the world, functions as a means to provide healthcare professionals with an objective justification into differentially diagnosing sets of clinical manifestations that patients' exhibit at one time or another. DXplain notates the likelihood of a disease by ranking the most probable at the top of the list and the least probable, yet still possible, at the bottom. DXplain further suggests what other clinical specifics would aid in further substantiating one disease from another and lists what clinical manifestations would be very much unusual for each of the generated diseases based on the originally inputted clinical predispositions of the patient.

DXplain initially began development in 1984 at the Massachusetts General Hospital Clinical and Research Informatics Division of the Department of Medicine and was released by 1986 with a knowledge base of five hundred diseases. National distribution of the system was not till 1987 consisting of a database containing approximately two thousand diseases and was delivered via dial-up until 1995. A standalone version of the system was distributed between 1991 and 1996 where individuals could load the version on any PC and at present the only means available in accessing the system, which replaced all older versions, is by way of the internet using a web-based version.

To date the knowledge base of DXplain includes over 2200 diseases and over 4900 clinical findings, which can consist of any of the following type of findings: symptoms, signs, epidemiological data, radiologic, endoscopic or laboratory findings, respectively. Each differential describes the relationship by representing the frequency with which the findings occur in the disease and by the degree to which the presence of the finding suggests a particular disease. Also, for each disease an associative value is presented within the system that provides a crude approximation of the disease prevalence by indicating whether the disease manifestation is common or rare and ranks accordingly to display all possibilities objectively.

Given the large knowledge base and accessible delivery of DXplain, the utilization of the program has come to support more than 33,000 healthcare professionals calculated in 2005 and has in turn assisted in formulating diagnostic hypotheses with healthcare providers toward patient health. The overall accuracy of Dxplain has since shown tremendous promise in citing the correct diagnosis in several instances. One of the first investigational trials conducted on the accuracy of DXplain consisted of forty-six modeled cases including a selection of different diseases to explain various clinical manifestations of each case. The ranked differential diagnoses generated by DXplain were no different than what a panel of five board-certified physicians diagnosed for each case. Moreover, several more recent studies do suggest that clinical decision support systems have the potential to minimize practice variation, improve patient care, and patient safety. DXplain, however, ought not to be substituted for physician diagnostic decision making, but rather used collaboratively to simply ensure an objective approach toward patient care where various health conditions a physician ought not to have sought will in fact do so with the use of the system.

DXplain recognizes an extensive amount of clinical findings, which include patient demographics, histories, physical exams, laboratory test results, and a range of specialty tests like X-rays, CTs, MRIs, ECGs, or endoscopic findings. In addition, previously diagnosed disease or conditions and indications of foreign travel, occupational work or medicinal use can also be entered into the clinical findings textbox as such findings are included in the appropriate disease or health condition profiles. Entering such clinical data into the system is seemingly simple; the healthcare provider needs only to type the findings into the textbox entitled clinical findings and for each finding entered he or she must separate each with the use of a comma or semicolon. To obtain the differential diagnosis based on these findings the healthcare professional must then press the submit button below the textbox where the system will continue to the next screen displaying all the diagnoses possible. Under certain circumstances healthcare providers may enter the wrong spelling or a word that is not an exact match to what is required for the system to recognize the finding and under such instances DXplain is equipped with a synonym matching tool that suggests alternatives to what is meant by the healthcare professional from the original entry.

After the initial findings are submitted and the list of potential diagnoses are displayed DXplain will then offer a set of findings that are selected on the basis of being potentially useful in refining the differential by presenting a list of signs or symptoms to the healthcare provider where he or she may check as either present, absent, or unknown. Furthermore, if one or two of the findings are deemed as more important than others within the case by the physician, a focus feature can be enabled by clicking on the checkboxes next to the clinical finding that is thought to be a crucial part of the case. DXplain will then rank only those diseases or conditions that are known to have such a clinical manifestation of such identified findings checked off by the healthcare professional. If a disease is presented where the healthcare provider wishes to either obtain more information or feels somewhat confused as to why a particular disease is listed in the differential, he or she may click on the disease and the system will display a $D x$ Description that provides both a depiction of the disease and a list of journal references that may aid in the understanding of the disease or condition. Also, under those instances where a healthcare provider is puzzled with a disease listed, he or she can click the Evidence of $D x$ button to observe each clinical finding entered that supports the disease to those which are entered that are not known to be a finding relevant toward the disease in question. Once all the pertinent findings are entered, revised, and felt exhausted by the healthcare professional, he or she will be presented a refined list of possible diseases or health 
conditions that can then be further investigated with additional tests if required or assessed for treatment options within the referenced material provided under the disease description.

DXplain availability is limited to hospitals, medical schools, and healthcare organizations and requires that the institute agrees to a no cost Institutional Evaluation License in order to gain access to the system. The license is merely an established contract between the organization wishing access and Massachusetts' General Hospital where the organization agrees to the terms of use outlined by the hospital. Once submitted to the hospital the organization will be granted access to the system via the internet and provided a username and password in order to access the program.

\section{Conclusion}

With use of a clinical decision support system, when an attending physician meets with a patient, he or she would be able to address the patient in the same context as he or she would prior to having implemented a clinical decision support system. The physician, as such, would ask for the symptoms and would note the signs exhibited by the patient. The physician would then advise the patient on the type of diagnostic tests that would aid in obtaining a diagnosis. Thereafter, the physician would access, and together with the patient would agree on the next step, whether to take blood, or grant a requisition for a CT or MRI, for example. After review of the diagnostic tests, the physician would turn to the clinical decision support system to assist in obtaining an accurate differential for the patient's signs and symptoms. Thenceforth, the physician would arrange with the patient to either provide treatment, or if still inconclusive would continue the differential on the basis of what the clinical decision support system recommends ensuring an accurate and safe diagnosis. Intervention timelines with use of the clinical decision support system will vary from case-to-case given that some cases may be more complex than others. Nonetheless, with successful implementation of the intervention, patient safety will be of the greatest priority in Canada's hospitals.

\section{References}

1. Baker GR, Norton P (2004) Patient Safety and Healthcare Error in the Canadian
Healthcare System: A Systematic Review and Analysis of Leading Practices in Canada with Reference to Key Initiatives Elsewhere. Health Canada.

2. Baker GR, Norton PG, Flintoft V, Blais R, Brown A, et al. (2004) The Canadian Adverse Events Study: the incidence of adverse events among hospital patients in Canada. CMAJ 170: 1678-1686.

3. Doyle DJ (2002) Errors of our ways. CMAJ 166: 221.

4. Canadian Health Association (2002) Patient Safety and Quality Care: Action Required Now to Address Adverse Events.

5. Jacobs S, O'Beirne M, Derfiingher LP, Vlach L, Rosser W, et al. (2007) Errors and adverse events in family medicine: developing and validating canadian taxonomy of errors. Can Fam Physician 53: 271-276.

6. Laura JMD (2005) First, Do No Harm: Medical Error in Canada. 1st Prize 2006 SDL Paper Award.

7. Porto GG (2001) Safety by Design: Ten Lessons from Human Factors Research. J Healthc Risk Manag 21: 43-50.

8. CIHI (2005) Hospital Trend in Canada. Canadian Institute of Health Information

9. Simmon-Morton DG, Simmon-Morton BG, Parcel GS, Bunker JF (1988) Influencing personal and environmental conditions for community health: A multilevel intervention model. Family and Community. The Journal of Health Promotion \& Maintenance 11: 25-35.

10. Wachter RM, McDonald KM (2001) Making Health Care Safer: A Critical Analysis of Patient Safety Practices. AHRQ Publication.

11. Munro, Margaret (2007) Men get better hospital care than women: report Ottawa Citizen.

12. Canadian Health Services Research Foundation (2004) Myth: We can eliminate errors in healthcare by getting rid of the "bad apples".

13. Sintchenko V, Coiera E, Iredell JR, Gilbert GL (2004) Comparative impact of guidelines, clinical data and decision support on prescribing decisions: an interactive web experiment with simulated cases. J Am Med Infor Assoc 11: 71-77.

14. Bates DW, Teich JM, Lee J, Seger D, Kuperman GJ, et al. (1999) The impact of computerized physician order entry on medication error prevention. J Am Med Inform Assoc 6: 313-321.

15. Bates DW, Cullen DJ, Laird N, Petersen LA, Small SD, et al. (1995) Incidence of adverse drug events and potential adverse drug events: Implications for prevention. JAMA 274: 29-34. 